\title{
Target mass corrections and beyond ${ }^{1}$
}

\author{
Alberto Accardi \\ Hampton University, Hampton, VA 23668, USA and Jefferson Lab, Newport News, VA 23606, USA
}

\begin{abstract}
I examine the uncertainty of perturbative QCD factorization for (un)polarized hadron structure functions in deep inelastic scattering at a large value of the Bjorken variable $x_{B}$. The focus will be on Target Mass Corrections and Jet Mass Corrections in the collinear factorization framework.
\end{abstract}

Keywords: polarized DIS, target mass corrections, collinear factorization, OPE

PACS: $12.38 . \mathrm{Bx}, 13.60 . \mathrm{Hb}$

Precise parton distribution functions (PDFs) at large parton fractional momentum $x$ are vital for understanding the nonperturbative structure of the nucleon, and the effects of color confinement on its partonic constituents. For instance, the $d / u$ quark distribution ratio near $x=1$ is very sensitive to the nature of the quark-quark forces in the nucleon, as are parton-parton correlations in the nucleon wave function. Moreover, the magnitude and sign of the ratios of spin-polarized to spin-averaged PDFs, $\Delta u / u$ and particularly $\Delta d / d$, in the limit $x \rightarrow 1$ reflect the nonperturbative quark-gluon dynamics in the nucleon, and can shed light on the origin of the nucleon's spin. Improved PDFs at large $x$ will be crucial at the upcoming $12 \mathrm{GeV}$ Jefferson Lab Upgrade for the planning and analysis of a host of experiments, as well as at upcoming neutrino oscillation experiments such as MINOS and MINER $v$ A at Fermilab to control and reduce systematic errors to a minimum. They will also directly impact experiments at hadron colliders such as the Large Hadron Collider at CERN, by facilitating accurate extraction of new physics signals as excess compared to benchmark perturbative QCD (pQCD) calculations, and by better constraining PDFs at lower $x$ and larger $Q^{2}$ via pQCD evolution.

Parton distribution functions can be extracted from experimental data through global QCD fits, which combine data from many different processes and observables, and analyze them by means of pQCD calculations. Currently, the unpolarized parton distributions are well-determined for $x \lesssim 0.5$ for quarks and $x \lesssim 0.3$ for gluons. To better constrain them at large $x$ it is necessary to study hard scattering processes near kinematic thresholds, such as Deep Inelastic Scattering (DIS) at large Bjorken invariant $x_{B}$ and low 4-momentum transfer squared $Q^{2}$, or Drell-Yan (DY) lepton pair production at large Feynman variable $x_{F}$. The large- $x$ and low- $Q^{2}$ region is even more important in the case of parton helicity and transversity distributions, which measure the parton's spin distribution and correlation to its orbital angular momentum, since much of the presently available experimental data lie in this kinematic domain.

Theoretical improvements in calculating leading-twist perturbative cross sections are needed in order to confidently access large- $x /$ low- $Q^{2}$ PDFs. In this talk, I use the

${ }^{1}$ Talk given at "SPIN2008”, University of Virginia, October 6-11 2008. 
collinear factorization framework to formulate and quantify corrections to DIS structure functions arising from target and jet mass corrections [1,2]. Threshold resummation, higher twists, quark-hadron duality in the pre-asymptotic domain, and nuclear effects such as binding, Fermi motion and nucleon off-shellness are left for future efforts.

Target mass corrections (TMC) for spin-averaged nucleon structure functions were first considered by Georgi \& Politzer within the Operator Product Expansion (OPE) [3, 4]. For spin-dependent scattering they were evaluated within the same OPE formalism in Refs. $[4,5]$. One of the limitations of the OPE formulation of TMCs is the so-called "threshold problem", in which the target mass corrected structure functions remain nonzero at $x_{B} \geq 1$. This arises from the failure to consistently incorporate the elastic threshold in moments of structure functions at finite $Q^{2}$, resulting in nonuniformity of the $Q^{2} \rightarrow \infty$ and $n \rightarrow \infty$ limits, where $n$ is the rank of the moment. As a consequence, after performing an inverse Mellin transform on the moments, the structure functions acquire incorrect support at large $x_{B}$ [6]. Various prescriptions have been considered to tame the unphysical behavior as $x_{B} \rightarrow 1$. However, these approaches are not unique and sometimes introduce additional complications.

An alternative approach, which avoids the threshold ambiguities from the outset, involves formulating TMCs directly in momentum space using the collinear factorization (CF) formalism [1]. A further advantage of this formalism is that it can be readily extended to processes such as semi-inclusive DIS, where the OPE is not available, and indeed to any other hard scattering process. Additional corrections to structure functions at large $x_{B}$, such as discussed in the introduction, can also be naturally incorporated together with TMCs. At any order in the strong coupling constant $\alpha_{s}$, and considering only massless partons, the $\mathrm{CF}$ target-mass corrected transverse and longitudinal structure functions, $F_{T}$ and $F_{L}$, read

$$
F_{T, L}\left(x_{B}, Q^{2}, M^{2}\right)=\sum_{f} \int_{\xi}^{\xi / x_{B}} \frac{d x}{x} h_{f \mid T, L}\left(\frac{\xi}{x}, Q^{2}\right) \varphi_{f}\left(x, Q^{2}\right),
$$

where $f$ is the parton flavor, $M$ the target's mass, $\xi$ the Nachtmann scaling variable,

$$
\xi=\frac{2 x_{B}}{1+\sqrt{1+\gamma^{2}}} \quad \gamma^{2}=4 x_{B}^{2} M^{2} / Q^{2}
$$

$h_{f}$ the perturbatively calculable coefficients, and $\varphi_{f}$ the unpolarized PDFs. The polarized $g_{1}$ structure function reads

$$
g_{1}\left(x_{B}, Q^{2}, M^{2}\right)=\frac{1}{1+\gamma^{2}} \sum_{f} \int_{\xi}^{\xi / x_{B}} \frac{d x}{x} g_{1, f}\left(\frac{\xi}{x}, Q^{2}\right) \Delta \varphi_{f}\left(x, Q^{2}\right),
$$

where $g_{1, f}$ are the perturbatively calculable coefficients, and $\Delta \varphi_{f}$ are the parton helicity distributions. The upper limit of integration $x \leq \xi / x_{B}$ is imposed by combining four-momentum and baryon number conservation in the kinematics of the handbag diagram. As a result, the structure functions are zero when $x_{B} \geq 1$, which solves the threshold problem. Note also that contrary to previous claims [7], $F_{T, L}\left(x_{B}\right) \neq F_{T, L}^{(0)}(\xi)$, and $g_{1}\left(x_{B}\right) \neq\left(1+\gamma^{2}\right)^{-1} g_{1}^{(0)}(\xi)$, where the superscript $(0)$ indicates "massless" structure functions computed with $M=0$, and the upper limit on the $x$ integration is $x \leq 1$. 

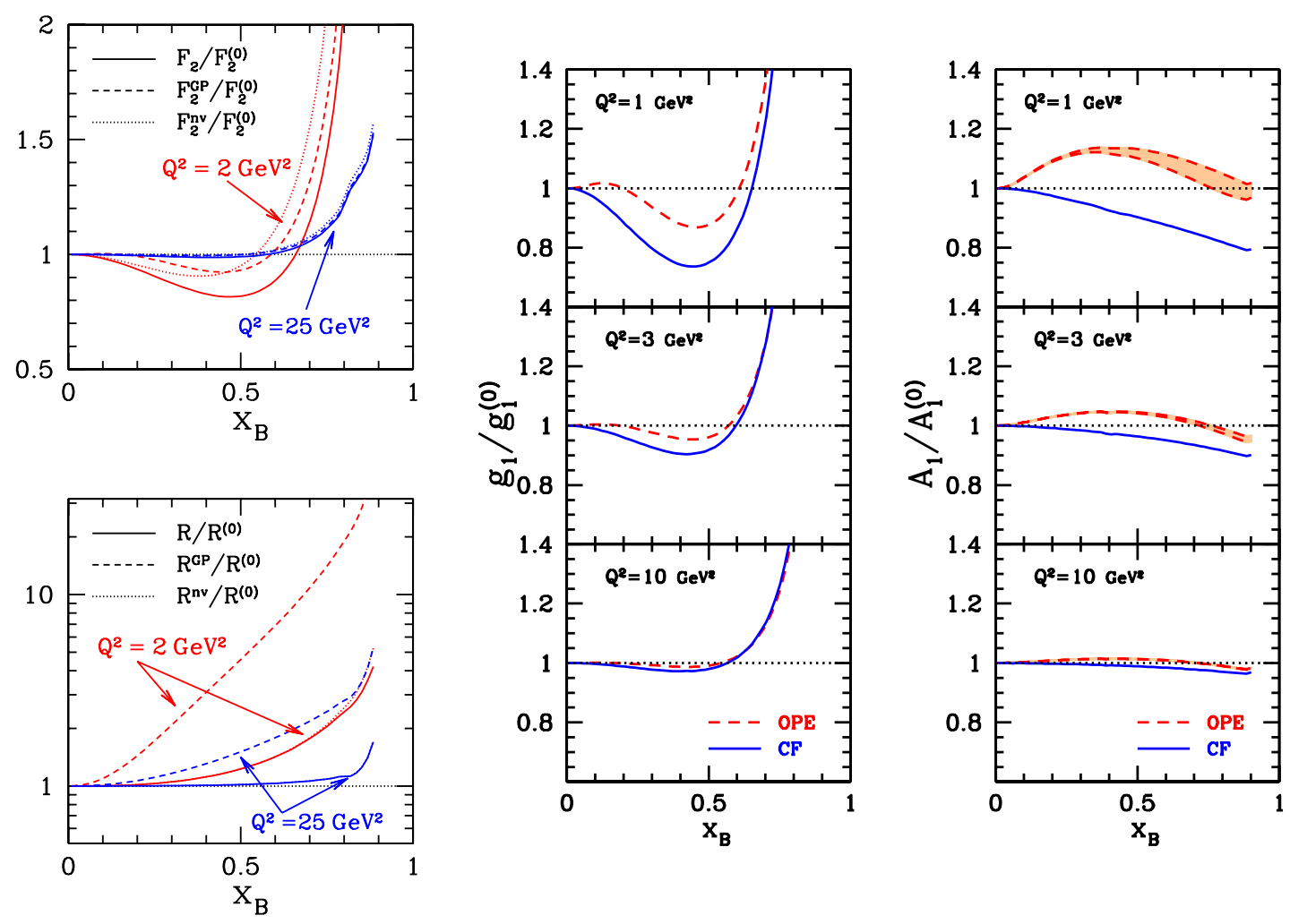

FIGURE 1. Square plots: Comparison of prescriptions for NLO target mass corrections to $F_{2}$ and $R=F_{L} / F_{1}$, computed with MRST2002 parton distributions [1]. (The "GP" curves are computed in OPE; the "nv" curves are computed using 1 as upper bound for the $x$ integration in Eq. (1). Vertical plots: Same for the LO $g_{1}$ structure function (left) and $A_{1}$ polarization asymmetry (right) [2]. For $A_{1}$, the shaded band for the OPE result indicates the uncertainty due to the use of the Wandzura-Wilczek relation (lower bound) or the identity $g_{1}+g_{2}=0$ (upper bound) in deriving $A_{1}$.

An estimate of TMC in collinear factorization for polarized and unpolarized structure functions, and for the $A_{1}=\left(1+\gamma^{2}\right) g_{1} / F_{1}$ asymmetry is presented in Fig. 1 , and compared to the TMC computed in OPE. The numerical results for the target mass corrections to the $F_{2}$ and $g_{1}$ structure functions are qualitatively similar in the CF and OPE computations. At leading order (LO), the differences of up to $30 \%$ for low $Q^{2}$ values disappear for $Q^{2} \gtrsim 10 \mathrm{GeV}^{2}$. At next-to-leading order (NLO), the differences are generally larger than at LO for intermediate $x_{B}$ values. In all cases the TMCs remain significant at $x_{B} \gtrsim 0.7$ even for $Q^{2}>10 \mathrm{GeV}^{2}$, and need to be taken into account when analyzing large- $x_{B}$ data. In the longitudinal $F_{L}$ structure function the CF and OPE corrections are larger by themselves, and differ by more than factors of several at large and not-so-large $x_{B}$, even at large $Q^{2}$ values usually considered TMC-free. The choice of TMC scheme will affect, e.g., the determination of the gluon PDF, to which $F_{L}$ is much more sensitive than $F_{2}$, and is not a negligible matter. Since the TMCs are qualitatively similar for the $g_{1}$ and $F_{1}$ structure functions, they largely cancel in the $A_{1}$ asymmetry, although the sign of the correction is opposite in the CF and OPE approaches over most of the range of $x_{B}$, but can be as large as $20 \%$ at low $Q^{2}$, disappearing at $Q^{2} \gtrsim 10 \mathrm{GeV}^{2}$. The commonly used approximation relating $A_{1}$ directly to the longitudinal asymmetry $A_{\|}$can be shown 
to be equivalent to assuming $A_{1} \approx A_{1}^{(0)}$ [2]. Fig. 1 shows that it breaks down at low $Q^{2}$ : an accurate measurement of $g_{1}$, e.g., at JLab, requires both $A_{\|}$and $A_{\perp}$ asymmetries.

Even after solving the threshold problem as described, the structure functions remain unphysically positive as $x_{B} \rightarrow 1$ because the assumption that at LO the final state current jet is made of a single massless quark makes the LO perturbative coefficients proportional to $\delta\left(x-\xi / x_{B}\right)$. Such an assumption is clearly unphysical because the quark has to hadronize due to color confinement, so that the current jet will have an invariant mass $m_{j}^{2}$. Using this mass in the kinematics of the LO handbag diagram, and assuming that the invariant jet mass has a probability distribution $J_{m}$, one obtains

$$
F_{T}^{J M C}\left(x_{B}, Q^{2}, M^{2}\right)=\int_{0}^{\frac{1-x_{B}}{x_{B}} Q^{2}} d m_{j}^{2} J_{m}\left(m_{j}^{2}\right) F_{T}^{(0)}\left(\xi\left(1+m_{j}^{2} / Q^{2}\right), Q^{2}\right) .
$$

If the "jet function" $J_{m}\left(m_{j}^{2}\right)$ is a sufficiently smooth function of $m_{j}^{2}$, one obtains $F_{T}^{J M C}\left(x_{B}, Q^{2}, M^{2}\right) \rightarrow 0$ as $x_{B} \rightarrow 1$, as it should be. The jet mass corrections (JMC) so introduced are of order $O\left(m_{j}^{2} / Q^{2}\right)$, and it is easy to see that in the limit $Q^{2} \gg\left\langle m_{j}^{2}\right\rangle$ one recovers the structure functions with TMC only. It turns out that the ansatz (4) is theoretically correct, and that the jet function is the spectral function, $J_{2}$, of a vacuum quark propagator,

$$
\int_{0}^{\infty} d m_{j}^{2} J_{2}\left(m_{j}^{2}\right) 2 \pi \delta\left(l^{2}-m_{j}^{2}\right) \theta\left(l^{0}\right)=\frac{1}{4 l^{-}} \int d^{4} z e^{i z \cdot l} \operatorname{Tr}\left[\gamma^{-}\langle 0|\bar{\psi}(z) \psi(0)| 0\rangle\right],
$$

additionally smeared by soft momentum exchanges with the target jet [1]. A toy model estimate of these Jet Mass Corrections shows that they may in fact be comparable in size to the TMC. However their phenomenology is just at its beginning.

\section{ACKNOWLEDGMENTS}

This work was supported by the DOE contract No. DE-AC05-06OR23177, under which Jefferson Science Associates, LLC operates Jefferson Lab, and NSF award No. 0653508.

\section{REFERENCES}

1. A. Accardi and J. W. Qiu, JHEP 0807 (2008) 090.

2. A. Accardi and W. Melnitchouk, Phys. Lett. B 670 (2008) 114.

3. H. Georgi and H. D. Politzer, Phys. Rev. D 14 (1976)1829; A. De Rujula, H. Georgi and H. D. Politzer, Annals Phys. 103 (1977) 315.

4. J. Blumlein and A. Tkabladze, Nucl. Phys. B 553 (1999) 427.

5. S. Matsuda and T. Uematsu, Nucl. Phys. B 168 (1980) 181; A. Piccione and G. Ridolfi, Nucl. Phys. B 513 (1998) 301; J. Blumlein and A. Tkabladze, Nucl. Phys. B 553 (1999) 427.

6. K. Bitar, P. W. Johnson and W. K. Tung, Phys. Lett. B 83 (1979) 114; P. W. Johnson and W. K. Tung, Print-79-1018 (Illinois Tech) Contribution to Neutrino '79, Bergen, Norway, June 18-22, 1979. For a review, see I. Schienbein et al., J. Phys. G 35 (2008) 053101.

7. M. A. G. Aivazis, F. I. Olness and W. K. Tung, Phys. Rev. D 50 (1994) 3085; S. Kretzer and M. H. Reno, Phys. Rev. D 69 (2004) 034002. 